\title{
Les ondes électromagnétiques térahertz au service de la sécurité et de la défense
}

Chercheurs et ingénieurs rêvent d'utiliser les ondes électromagnétiques térahertz (THz), c'est-à-dire appartenant à l'infrarouge très lointain, dans de nombreuses applications entrevues dans des domaines aussi variés que les télécommunications à très haut débit, l'imagerie médicale, les capteurs pour l'industrie, etc., parmi lesquels la sécurité et la défense prennent une part prépondérante. Les activités de recherche et développement dans le domaine THz ont été stimulées par l'avènement, à la fin des années 1980, de nouvelles techniques et technologies, basées principalement sur l'optoélectronique, et actuellement mettant aussi en jeu la micro- et la nanoélectronique. Cet article présente les principes de la technologie THz [1] et l'état de l'art des études et développements pour la sécurité et la défense. Cet état de l'art est sans doute incomplet, car nombre des travaux menés sont classés confidentiels et ne sont pas publiés.

\author{
Jean-Louis COUTAZ \\ Frédéric GARET \\ IMEP-LAHC, UMR 5130 du CNRS \\ Université de Savoie \\ coutaz@univ-savoie.fr
}

\section{Physique des ondes $\mathrm{THz}$}

Le domaine $\mathrm{THz}$ s'étend typiquement depuis les fréquences de l'ordre de la centaine de $\mathrm{GHz}$ jusqu'à quelques dizaines de $\mathrm{THz}$, c'est-à-dire qu'il englobe les longueurs d'onde de quelques dizaines de microns à quelques millimètres (figure 1). Il est donc situé entre l'optique (infrarouge) et l'électronique (hyperfréquences). L'énergie des photons $\mathrm{THz}$ étant très faible (quelques meV), le rayonnement $\mathrm{THz}$ n'excitera dans la matière que des résonances peu énergétiques, telles les vibrations globales des structures moléculaires (figure 2), les phonons optiques dans les solides, les porteurs libres, les paires de Cooper dans les supraconducteurs, etc. En conséquence, un grand nombre de matériaux, par exemple les diélectriques ou les semi-conducteurs intrinsèques, sont transparents pour les rayons $\mathrm{THz}$.

Les métaux présentent une conductivité pratiquement infinie, et se comportent donc comme des métaux parfaits dont la réponse électromagnétique est décrite par la théorie de Drude.
Les molécules, surtout polaires, peuvent présenter des spectres d'absorption rotovibrationnels plus ou moins complexes dans cette gamme fréquentielle. Citons deux exemples très importants. Tout d'abord, la molécule d'eau, qui montre des raies d'absorption intenses de plus en plus nombreuses à partir de $500 \mathrm{GHz}$. Cela se traduit par une opacité de l'air au voisinage de ces raies, à cause de la vapeur d'eau (figure 3). De même, les ondes $\mathrm{THz}$ ne se propagent pas dans l'eau liquide et dans les milieux aqueux ou humides comme le corps humain. Typiquement, la profondeur de pénétration d'une onde $\mathrm{THz}$ dans l'eau ou dans un tissu biologique est l'ordre de la centaine de microns. Deuxième exemple, les grosses molécules organiques - aliments, explosifs (voir figure 4b), médicaments, etc. - qui exhibent une signature spectrale caractéristique dans le domaine $\mathrm{THz}$. Ces deux exemples indiquent les directions des recherches applicatives menées aujourd'hui : d'une part, l'imagerie, basée sur le degré de transparence des matériaux ; d'autre part, la détermination de la composition chimique d'échantillons par analyse spectrale. Bien sûr, on peut combiner les deux en vue de l'imagerie hyperspectrale $\mathrm{THz}$, dont nous décrirons l'intérêt plus loin.

\section{Technologie des ondes $\mathrm{THz}$}

Depuis le début du $x x^{\mathrm{e}}$ siècle jusqu'aux années 1990, l'infrarouge lointain a été remarquablement étudié, par exemple en France, à Nancy, par A. Hadni, mais au prix de travaux fastidieux liés à l'absence de sources et détecteurs performants ou pratiques. Le rayonnement était essentiellement produit par des corps noirs, comme les lampes à mercure, et les détecteurs étaient des bolomètres ou des cellules de Golay, dont respectivement la résistance et le volume varient avec l'augmentation de température induite par l'absorption du rayonnement. La faible brillance des sources et

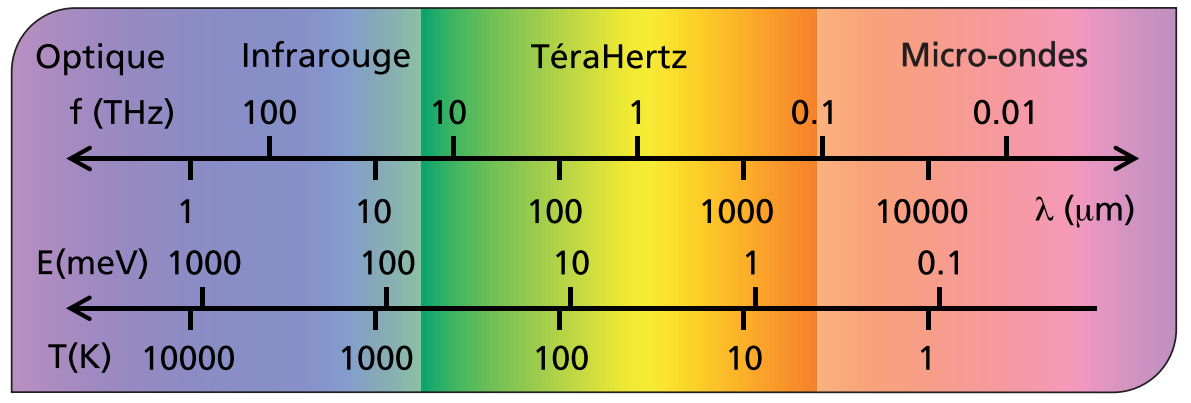

Figure 1. Le domaine $\mathrm{THz}$ au sein du spectre électromagnétique. 


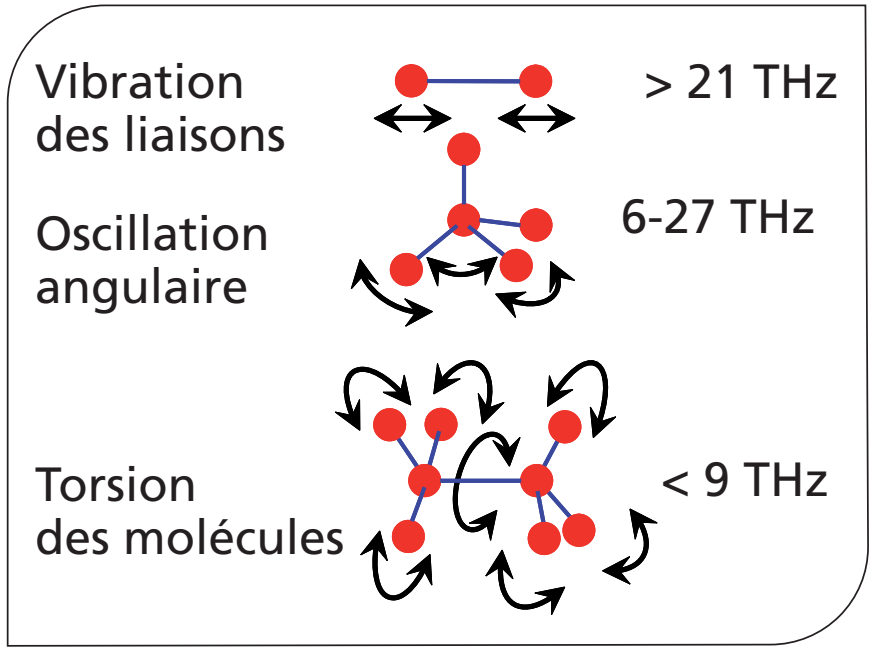

Figure 2. Domaines de fréquence des résonances moléculaires.

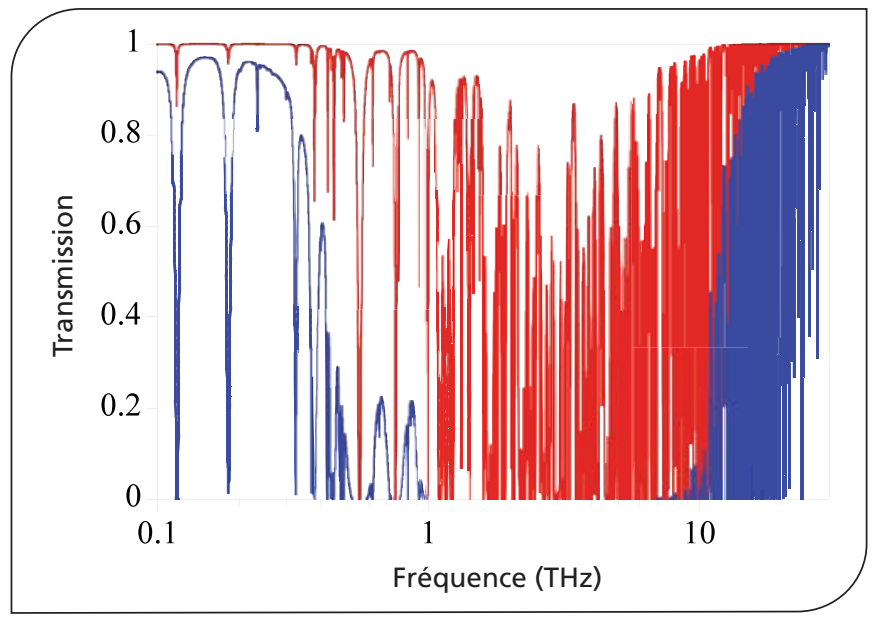

Figure 3. Transmission de l'atmosphère (en rouge épaisseur $1 \mathrm{~m}$, en bleu $100 \mathrm{~m}$ ) dans le domaine THz. leur incohérence conduisaient à des enregistrements de spectre pouvant durer une semaine! Les lasers moléculaires ou les tubes électroniques (carcinotron) étaient employés dans des cas particuliers. L'avènement des lasers femtosecondes a révolutionné cette science. En effet, le redressement optique des impulsions lasers permet de générer des bouffées électromagnétiques dont la forme est typiquement l'enveloppe des impulsions optiques, élargie et déformée par la dispersion. Typiquement, ce signal électromagnétique a une durée sub-picoseconde, donc un spectre de largeur THz, puisqu'inversement proportionnel à la durée des impulsions. Ainsi, le redressement optique permet de transposer les fréquences optiques de l'impulsion laser au domaine $\mathrm{THz}$. $\mathrm{Ce}$ redressement est réalisé soit dans des cristaux non linéaires, comme $\mathrm{ZnTe}$, soit dans des semi-conducteurs, où les porteurs photogénérés présentent une durée de vie sub-picoseconde (par exemple GaAs épitaxié à basse température). La mesure de ce signal THz est effectuée par des techniques d'échantillonnage grâce à la périodicité du train d'impulsions laser, le signal THz étant en fait mélangé avec l'impulsion optique initiale, retardée par une ligne à retard, dans des dispositifs assez semblables aux émetteurs.

Ces techniques optoélectroniques évoluent aujourd'hui dans deux directions : d'une part des systèmes compacts et efficaces, si possible utilisant les lasers et les composants des télécommunications pour réduire les coûts; d'autre part des systèmes délivrant des signaux $\mathrm{THz}$ continus, basés sur le battement optique de deux lasers de longueurs d'onde légèrement différentes.

Vers les années 2000, d'autres composants sont apparus ou sont devenus compétitifs. Parmi les sources, les lasers à cascade quantique sont très prometteurs, mais la faible énergie des photons $\mathrm{THz}$, inférieure à l'énergie thermique à température ambiante, les oblige à fonctionner à des températures cryogéniques. Les sources purement électroniques ont réalisé beaucoup de progrès et $\gg$

\section{SPECTROGON}

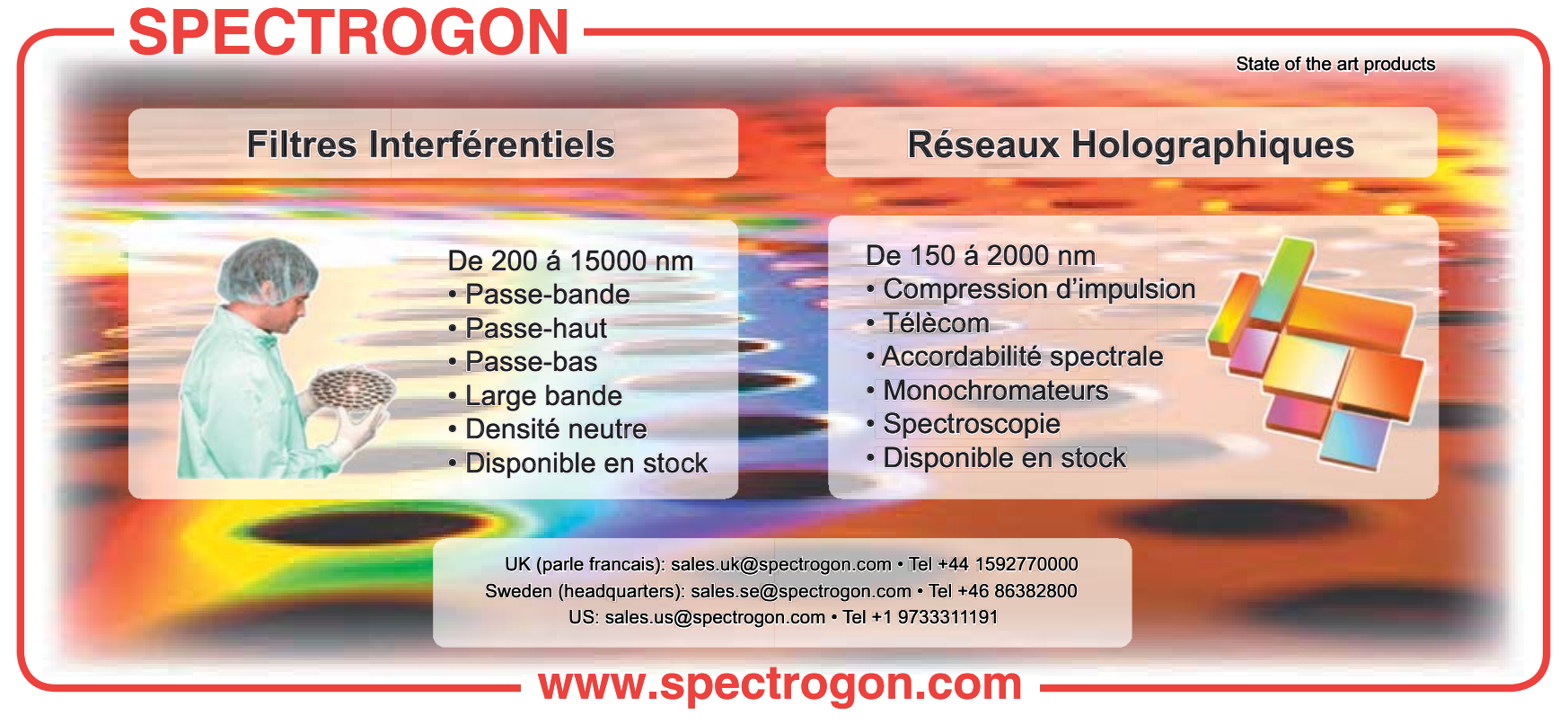



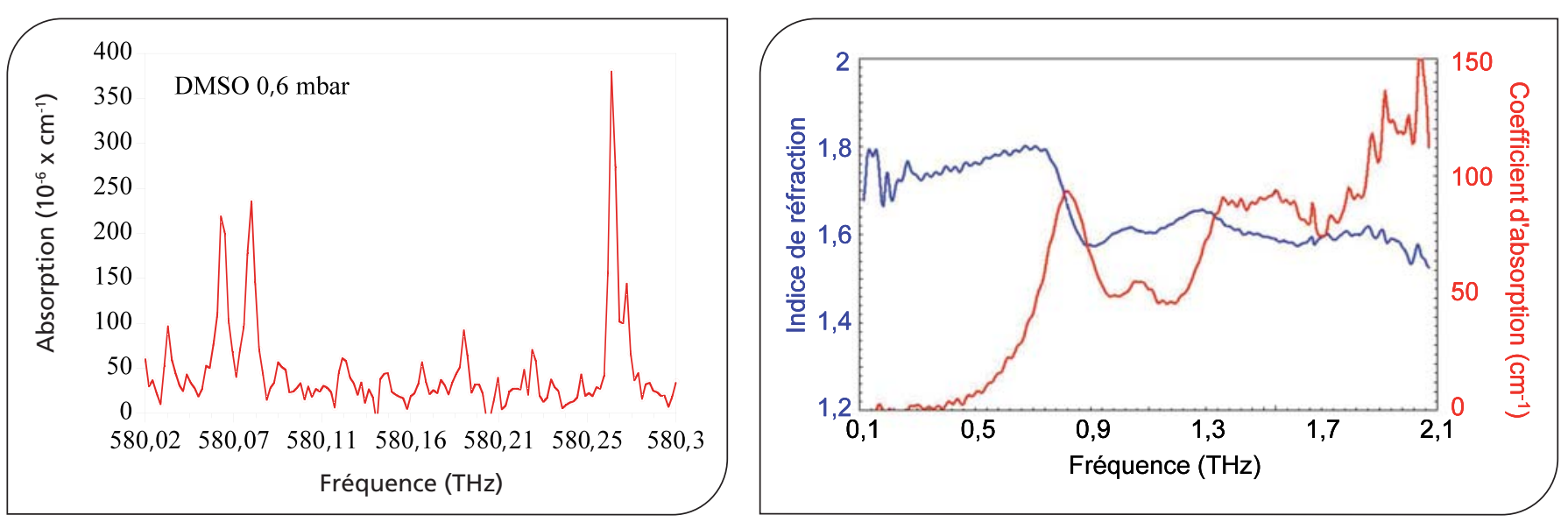

Figure 4. Spectres d'absorption (à gauche) de la molécule DMSO mesurés par F. Hindle (LPCA-Dunkerque) [2], (à droite) de l'explosif RDX (Université de Savoie).

surpasseront sans doute les sources optiques pour les «basses» fréquences, c'est-à-dire jusqu'à $500-700 \mathrm{GHz}$. Ce sont par exemple des diodes Schottky qui multiplient en fréquence un signal à quelques dizaines de $\mathrm{GHz}$ délivré par une source hyperfréquence. Les composants de la nanoélectronique sont de plus en plus nombreux, comme les transistors FET à canal nanométrique dans lequel le gaz électronique bidimensionnel oscille à sa fréquence plasma située dans le domaine THz. Néanmoins, il faut admettre qu'aujourd'hui aucune source n'est suffisamment puissante, efficace, compacte ou simple d'emploi pour permettre le développement immédiat des applications grand public de la technologie THz. Simultanément, les détecteurs deviennent de plus en plus performants. Ils sont caractérisés par la puissance $\mathrm{THz}$ détectable minimale, exprimée en puissance équivalente de bruit normalisée à la bande spectrale (NEP en W.Hz ${ }^{-1 / 2}$ ). Les détecteurs cryogéniques sont aujourd'hui très aboutis. Ainsi, ceux à supraconducteurs, dans lesquels les photons $\mathrm{THz}$ brisent des paires de Cooper pour former un gaz d'électrons chauds qui augmente la résistance du dispositif, atteignent un NEP de $10^{-16} \mathrm{~W} . \mathrm{Hz}^{-1 / 2}$, largement suffisant pour détecter le signal $\mathrm{THz}$ du rayonnement de corps noir d'un individu. Ces bolomètres sont très utilisés en radioastronomie et peuvent être construits sous forme matricielle pour l'imagerie.
Encore plus spectaculaires, les transistors FET sur lesquels une boîte quantique en semi-conducteur dopé, servant de grille pour le transistor, voit son gaz d'électrons osciller à la fréquence plasma sous éclairement $\mathrm{THz}$ et alimente ainsi le canal du transistor. Ces transistors permettent pratiquement la détection de photon $\mathrm{THz}$ unique, mais à des températures de l'ordre du Kelvin. Les détecteurs à température ambiante sont très étudiés pour les applications grand public, mais leur développement est rendu difficile par la nécessité d'isoler le signal du bruit thermique ambiant. Citons comme dispositifs prometteurs les microbolomètres à membrane dérivés de ceux utilisés dans l'infrarouge, et aussi les nanotransistors à effet plasma décrits plus haut. Des premiers films vidéo $\mathrm{THz}$ ont été tournés en laboratoire, mais en éclairant la scène avec un laser $\mathrm{THz}$ puissant. Comme pour les émetteurs, un effort technologique important est nécessaire pour disposer de détecteurs performants.

\section{Applications de la technologie $\mathrm{THz}$ aux problèmes de sécurité et défense}

\section{Détection de gaz et agents létaux}

De nombreux gaz, formés de molécules suffisamment grosses, présentent un spectre d'absorption typique dans le domaine $\mathrm{THz}$, alors qu'ils sont pratiquement transparents dans le visible et dans l'infrarouge. Un exemple bien connu est le disulfure d'hydrogène, à l'odeur d'œuf pourri caractéristique, mais on peut citer aussi l'ozone, etc. Cela ouvre la voie à la détection à distance par spectroscopie d'absorption THz de gaz polluants dans l'atmosphère, à la réalisation de capteurs pour l'industrie, etc. Dans le domaine de la sécurité et de la défense, on cherche à détecter à distance des gaz ou des agents chimiques et biologiques létaux, que ce soit sur un champ de bataille lors de conflits, ou bien dans des zones fréquentées par la population, dans le cas de problèmes liés au terrorisme. Pour cela, il faut d'abord s'assurer que la substance recherchée présente une signature spectrale dans le domaine THz. C'est le cas des gaz de combat, comme les trop célèbres gaz moutarde et sarin. La figure $4 a$ montre le spectre du diméthyle sulfoxide (DMSO) [2], qui ressemble fortement à celui du gaz moutarde, et permet de mener en laboratoire des études de faisabilité sans être exposé au danger du gaz moutarde. De même, le spectre des agents biologiques comme l'anthrax ou son substitut Bacillus subtilis, sont bien identifiables au-dessus de $1 \mathrm{THz}$ [3]. Pour détecter la présence de ces substances, si possible à quelques dizaines ou centaines de mètres, il faut ensuite construire un appareil qui permet d'illuminer la scène avec un faisceau $\mathrm{THz}$, puis qui mesure le signal $\mathrm{THz}$ réfléchi à l'endroit du gaz à détecter. Le challenge technologique est difficile, car la plupart des substances à détecter présentent 
un spectre intéressant dans la gamme des quelques $\mathrm{THz}$, pour laquelle l'air est très absorbant. Une solution élégante est proposée et développée par X.-C. Zhang (Institut Rennselaer, Troy, États-Unis). Le signal $\mathrm{THz}$ est généré et mesuré à l'endroit où se trouve le gaz, en faisant claquer dans l'air une impulsion laser femtoseconde intense. L'air se comporte comme un milieu optique non linéaire qui redresse le signal optique, et permet la mesure du champ THz par effet Kerr. L'information THz est transportée par le faisceau optique, qui ne souffre pas de l'atténuation de l'air.

\section{Principes et bases de l'imagerie $\mathrm{THz}$}

Dans les domaines de l'infrarouge et des $\mathrm{THz}$, il existe deux familles d'imagerie. L'imagerie passive profite du rayonnement thermique émis par un objet à température non nulle. Lorsque ce rayonnement est trop faible ou bien est noyé dans un bruit de fond ambiant, il est nécessaire d'éclairer la scène avec un faisceau THz (imagerie active). Dans le domaine THz, la loi de Planck de rayonnement du corps noir est approximée par celle de Rayleigh-Jeans. Pour un système optique d'imagerie passive limité par la diffraction, c'est-à-dire tel que la plus petite zone résolue sur la scène correspond dans le plan image à un pixel du détecteur, on démontre que la puissance reçue par le pixel est $2 k_{R} T \Delta v$, où $T$ est la température du corps rayonnant, $k_{B}$ la constante de Boltzmann et $\Delta v$ la bande passante du détecteur. La réalisation d'une image nécessite un objet avec des températures différentes, l'image correspondant en fait à la cartographie thermique de l'objet puisque la formule précédente ne dépend pas de l'émissivité de l'objet. On définit alors la température équivalente moyenne de bruit (NEDT en anglais), qui est la plus petite variation de température que le système optique peut visualiser. En dérivant la formule précédente, on aboutit à NEDT $=\Delta T \min =\mathrm{NEP} / 2 k_{B} \Delta v$.

La température d'un objet dissimulé sous les vêtements d'une personne diffère de quelques dixièmes de degrés de celles de la peau ou des vêtements (figure 5). Pour un détecteur avec une bande passante de $100 \mathrm{GHz}$, le NEP requis est de l'ordre de $10^{-13} \mathrm{~W} \cdot \mathrm{Hz}^{-1 / 2}$. Aujourd'hui, seuls les détecteurs cryogéniques atteignent cette sensibilité. Les autres détecteurs, dont les bolomètres à température ambiante, demandent que la scène soit éclairée, ce qui pose deux problèmes pour les applications grand public : I'absence de sources $\mathrm{THz}$ performantes et la crainte du public d'être éclairé par un rayonnement électromagnétique. Ce dernier point n'est pas déterminant quand il s'agit d'examiner des objets suspects, par exemple des enveloppes de courrier qui contiendraient des substances dangereuses. Notons enfin que la résolution spatiale de l'imagerie $\mathrm{THz}$ est de l'ordre du millimètre, limitée par la diffraction mais aussi par les aberrations des optiques, qui sont réfractives (composants et lentilles en polyéthylène, téflon ou silicium intrinsèque), ou réflectives (miroirs métalliques), ou même $\gg$

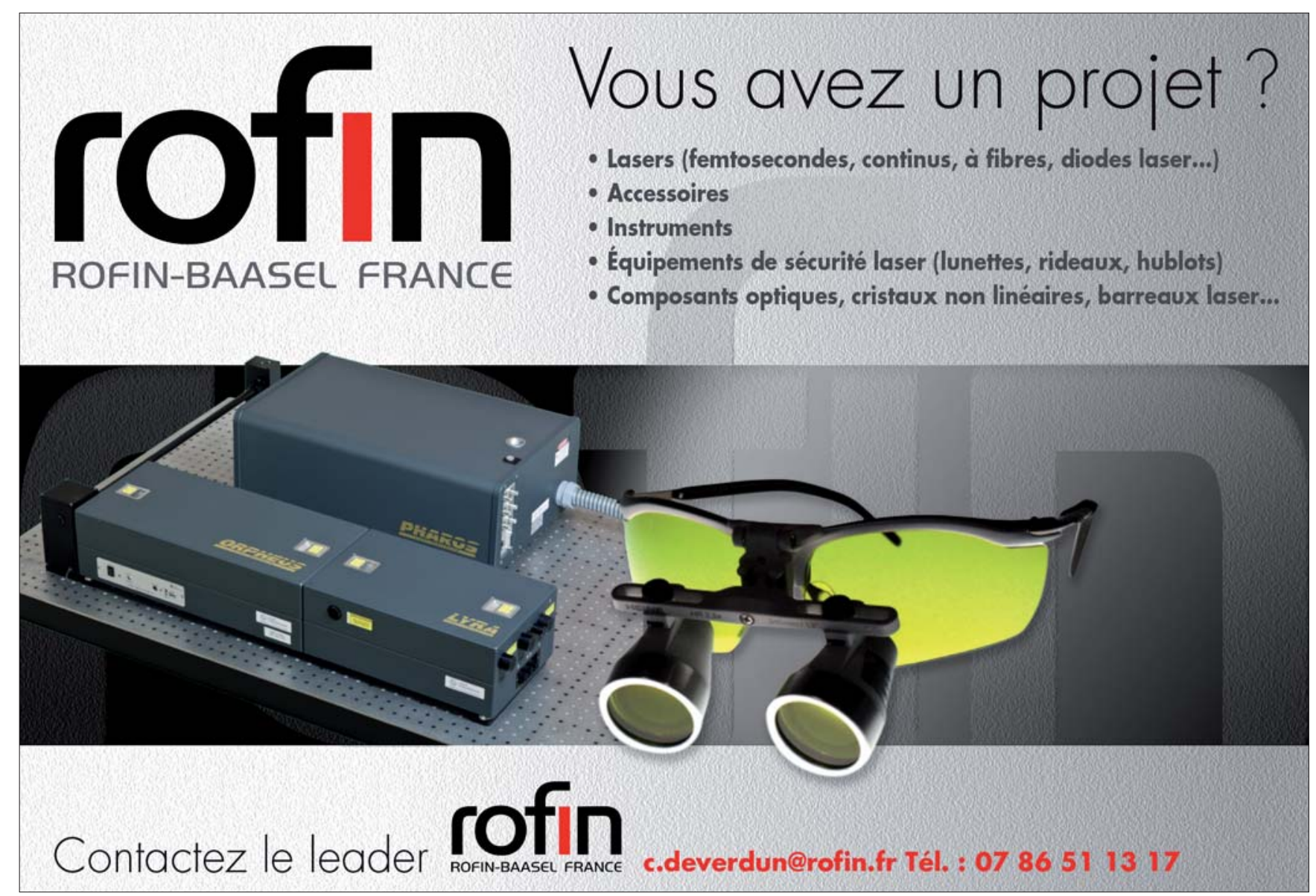


diffractives (lentilles de Fresnel ou lentilles métalliques, dont le fonctionnements'approche de celuides antennes en hyperfréquences).

\section{Détection d'objets dissimulés}

Les papiers, cartons, et tissus, s'ils sont secs, sont relativement transparents pour les ondes $\mathrm{THz}$. Une caméra $\mathrm{THz}$ peut ainsi facilement « voir » à travers les vêtements d'un individu, c'est-à-dire qu'elle le déshabille, posant dans certains pays des problèmes d'éthique compliqués et aussi une forte opposition de la population à ces techniques d'inspection. L'Union européenne a d'ailleurs publié en mai dernier une réglementation de 30 pages sur ce point [4] ! Les appareils installés, par exemple dans certains aéroports, fonctionnent à des fréquences de $30 \mathrm{ou}$ $90 \mathrm{GHz}$, car la technologie de ces fréquences est mature et permet d'obtenir des images exploitables, même si leur résolution est limitée à quelques centimètres. En laboratoire, des prototypes fonctionnent à plusieurs centaines de $\mathrm{GHz}$. L'observation d'objets métalliques (armes) ou de sachets de poudre est facile, d'autres matériaux étant moins « visibles".

Il faut bien noter que la technologie $\mathrm{THz}$ ne peut, seule, résoudre tous les problèmes de sécurité dans les portiques d'aéroport, et qu'elle ne s'envisage qu'associée à d'autres méthodes d'investigation. Ainsi, si les vêtements d'un individu sont mouillés, la caméra THz ne filmera pas les objets dissimulés. Mais le fait que les vêtements soient mouillés constitue un indice pour le personnel de sécurité, qui isolera l'individu suspect. L'examen d'objets pose moins de problème que celui des individus. Ainsi, un système $\mathrm{THz}$ automatisé d'inspection du courrier est installé dans des centres de tri postal au Japon (dont les noms ne sont pas révélés pour des raisons de sécurité). Chaque lettre ou colis postal est imagé à très grande vitesse par un système combinant une caméra visible, un appareil à rayons $\mathrm{X}$ et un détecteur à $90 \mathrm{GHz}$. Si l'objet semble suspect, il

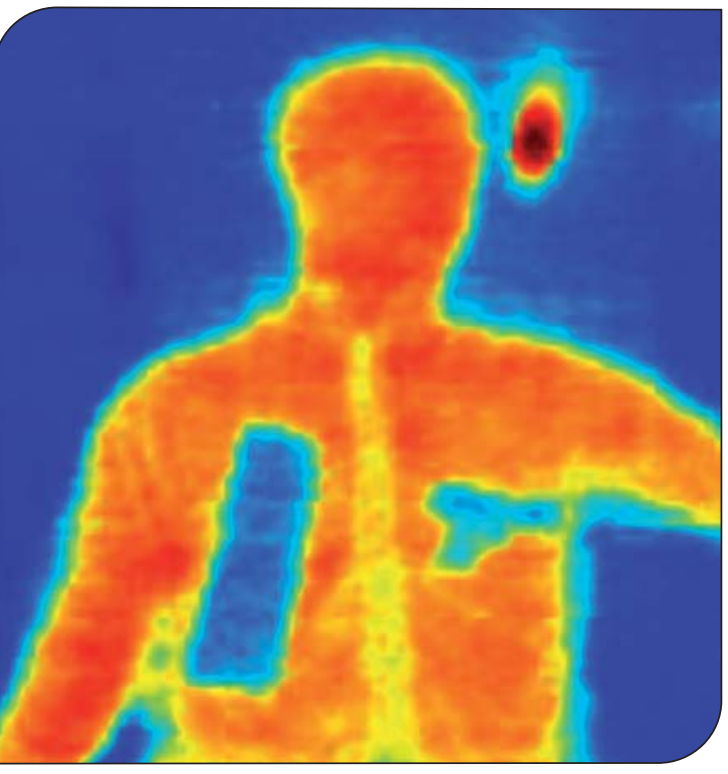

Figure 5. Image THz d'un individu dissimulant des objets suspects sous ses vêtements (cliché Arttu Luukanen, Millilab, Espoo, Finlande).

est isolé et inspecté plus lentement par un spectromètre $\mathrm{THz}$. Notons aussi que l'imagerie $\mathrm{THz}$ est assez résolue pour permettre la lecture à travers une enveloppe, car l'encre est plus absorbante que le papier. De la même façon, on peut " voir » des objets à travers une cloison, si celle-ci est par exemple constituée d'un matériau homogène de faible granulométrie comme le plâtre. Le béton, les briques ou d'autres matériaux présentent une granulométrie de dimension

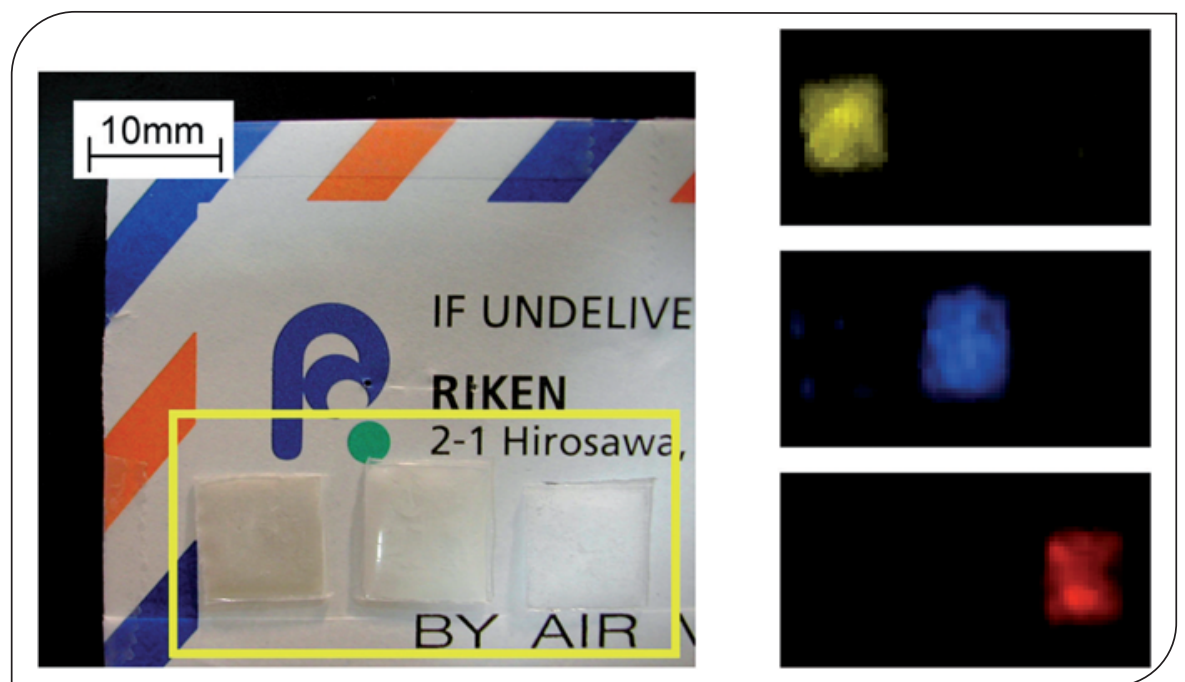

Figure 6. Reconnaissance de poudres cachées dans une enveloppe. À gauche, photographie de l'enveloppe et des sachets plastiques (de gauche à droite, dans l'encadré jaune : MDA, aspirine et méthamphétanime) ; à droite, images THz à différentes fréquences (fausses couleurs) permettant d'identifier les trois poudres (cliché Kodo Kawase, Université de Nagoya, Japon). 
mètres, le premier problème à résoudre est l'atténuation de l'air. C'est un problème difficile en l'absence actuelle de source puissante. La transparence de I'air diminue avec la fréquence, alors que les signatures spectrales des explosifs sont significatives à plusieurs $\mathrm{THz}$. On estime aujourd'hui que les futurs systèmes, si on arrive à les mettre au point, seront limités à l'observation à une dizaine de mètres au maximum. Le second problème est lié aux mesures qui sont souvent en mode de réflexion. II faut employer des optiques de large dimension, comme des télescopes, pour recueillir le maximum de signal qui est souvent diffusé par la rugosité des matériaux dans des directions non spéculaires.

De façon générale, les systèmes en développement aujourd'hui sont basés sur l'enregistrement du signal à quelques longueurs différentes et l'identification du produit par comparaison des mesures avec des bases de données. Les difficultés sont d'une part de trou- ver le nombre de longueurs d'onde nécessaires pour un taux d'identification élevé, nombre qui doit rester relativement faible pour des raisons de disponibilité des sources $\mathrm{THz}$, et d'autre part d'éviter les erreurs liées à des spectres proches pour des produits ou surtout des mélanges de produits très différents. Par exemple, l'explosif Metabel et le chocolat apparaissent identiques dans les scanners à rayons $X$ d'inspection des bagages. Leurs spectres $\mathrm{THz}$ sont aussi assez proches, mais un imageur THz sensible permet néanmoins de les identifier.

\section{Télécoms}

La transmission de signaux à l'aide d'une porteuse dans le domaine $\mathrm{THz}$ peut aussi être classée dans les technologies liées à la défense et sécurité. En effet, comme nous l'avons déjà expliqué, la propagation des ondes $\mathrm{THz}$ est limitée à quelques dizaines de mètres en atmosphère libre, ou à l'intérieur d'une pièce au sein d'un bâtiment.
Le signal ne pourra donc pas être détecté à plus longue distance ou dans une pièce voisine. On peut donc contrôler à qui il est diffusé. De plus, en tout cas à moyen terme, cette haute technologie n'est pas répandue et donc n'est pas disponible pour des groupes terroristes ou des pays moins développés.

\section{Références}

[1] Optoélectronique térahertz, édité sous la direction de J.-L. Coutaz, EDP Sciences, Paris (2008).

[2] F. Hindle et al. Recent Developments of an OptoElectronic $\mathrm{THz}$ Spectrometer for High-Resolution Spectroscopy. Sensors 2009; 9: 9039-9057.

[3] D. Woolard et al. Terahertz electronics for chemical and biological warfare agent detection. In IEEE MTT-S Int. Microwave Symp. Dig. Anaheim, CA, June 13-19, 1999 : 925-928.

[4] Rapport sur la sûreté aérienne, en particulier sur les scanners de sûreté, (2010/2154(INI)), Commission des transports et du tourisme, Communauté européenne, (Rapporteur : Luis de Grandes Pascual).

[5] K. Kawase, Y. Ogawa, and Y. Watanabe. Nondestructive terahertz imaging of illicit drugs using spectral fingerprints. Opt. Exp. 2003; 11, 2549.

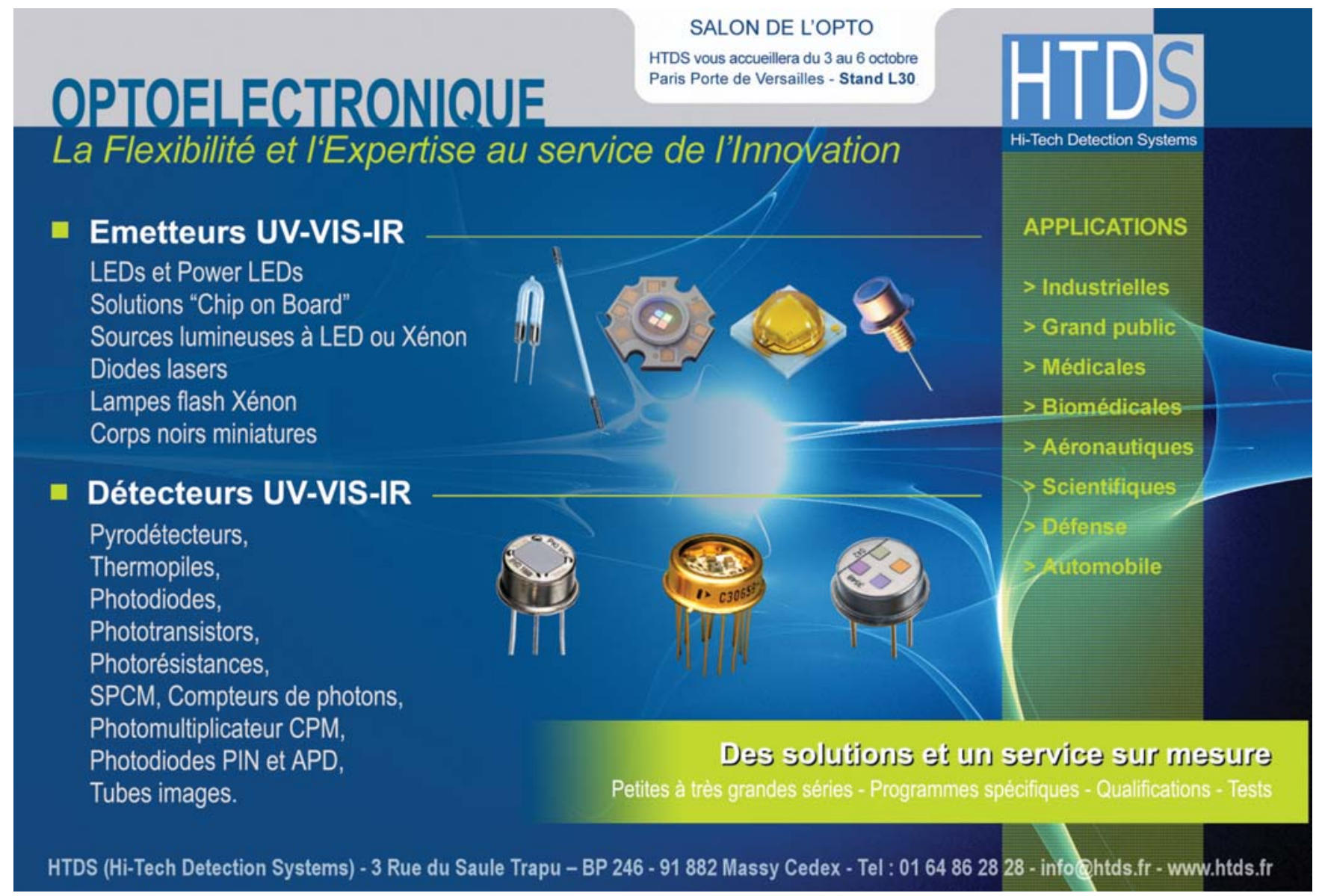

